\title{
Cosmology within Noncommutative Spectral Geometry
}

\section{Mairi Sakellariadou*}

Department of Physics, King's College, University of London, Strand WC2R 2LS, London, U.K.

E-mail: Mairi.Sakellariadou@kcl.ac.uk

Close to the Planck energy scale, the quantum nature of space-time reveals itself and all forces, including gravity, should be unified so that all interactions correspond to just one underlying symmetry. In the absence of a full quantum gravity theory, one may follow an effective approach and consider space-time as the product of a four-dimensional continuum compact Riemanian manifold by a tiny discrete finite noncommutative space. Since all available data are of a spectral nature, one may argue that it is more appropriate to apply the spectral action principle in this almost commutative space. Following this procedure one obtains an elegant geometric explanation for the most successful particle physics model, namely the standard model (and supersymmetric extensions) of electroweak and strong interactions in all its details, as determined by experimental data. Moreover, since this gravitational theory lives by construction at very high energy scales, it offers a perfect framework to address some of the early universe cosmological questions still awaiting for an answer.

After introducing some of the main mathematical elements of noncommutative spectral geometry, I will discuss various cosmological and phenomenological consequences of this theory, focusing in particular on constraints imposed on the gravitational sector of the theory.

Corfu Summer Institute on Elementary Particles and Physics - Workshop on Non Commutative Field Theory and Gravity,

September 8-12, 2010

Corfu Greece

\footnotetext{
* Speaker.
} 


\section{Introduction}

At energies much below the Planck scale, gravity can be considered as a classical theory, however as energies approach the Planck scale, the quantum nature of space-time becomes apparent, and the simple prescription, dictating that physics can be described by the sum of the EinsteinHilbert and the Standard Model (SM) action ceases to be valid. At such high energy scales, all forces, including gravity, are expected to be unified so that all interactions correspond to one underlying symmetry. Thus, near Planckian energies, the appropriate formulation of geometry should be within a quantum framework, while the nature of space-time would change in such a way so that one can recover the low energy picture of diffeomorphism and internal gauge symmetries, which govern General Relativity (GR) and gauge groups on which the Standard Model is based, respectively. A promising attempt to obtain a quantum nature of space-time has been realised within the realm of NonCommutative Geometry (NCG).

Noncommutative Geometry $[1,2]$ is a beautiful and rich mathematical theory, according which geometry can be described through the functions defined on the geometry, while the geometric properties of spaces can be described by the properties of functions defined on the spaces. An important new feature of noncommutative geometry is the existence of inner fluctuations of the metric, which correspond to the subgroup of inner automorphisms. Besides the mathematical beauty of NCG, which by itself explains why one may want to study this theory, NCG offers a variety of phenomenological consequences, which turn this theory into a fertile framework to address fundamental issues of early universe cosmology and high energy physics phenomenology.

In what follows we will follow Connes' approach [1,2] and consider a model of a two-sheeted space made from the product of a continuous space by a discrete space. This model led to a geometric explanation of the Standard Model; in particular the model shows that the vacuum Expectation Value of the Higgs field is related to the noncommutative distance between the two sheets. Within Connes' model of NCG, the Higgs field is conformally coupled to the Ricci curvature, while the generalised Einstein-Hilbert action contains in addition a minimally coupled massless scalar field related to the distance between the two sheets. Connes' approach is based upon a spectral action principle, stating that the bare bosonic Euclidean action for any noncommutative model based on a product (noncommutative) space is the trace of the heat kernel associated with the square of the noncommutative Dirac operator of the product geometry.

Within noncommutative spectral geometry, we look for a hidden structure in the functional of gravity coupled to the SM at today's low energy scales, and avoid an extrapolation by many orders of magnitude to guess the appropriate structure of space-time at Planckian energy scales.

Noncommutative spectral geometry offers an elegant approach to unification, based on the symplectic unitary group in Hilbert space, rather than on finite dimensional Lie groups. The model offers a unification of internal symmetries with the gravitational ones. All symmetries arise as automorphisms of the noncommutative algebra of coordinates on a product geometry. Due to the lack of a full quantum gravity theory, which a priori should define the geometry of spacetime at Planckian energy scales, we will follow an effective theory approach and consider the simplest case beyond commutative spaces. Thus, below but close to the Planck energy scale, spacetime will be considered as the product of a Riemanian spin manifold by a finite noncommutative space. At higher energy scales, space-time should become noncommutative in a nontrivial way, 
while at energies above the Planck scale the whole concept of geometry may altogether become meaningless. As a next, but highly nontrivial, step one should consider noncommutative spaces whose limit is the almost commutative space considered here. Unfortunately, the birth of geometry may remain an unsolved puzzle for still quite sometime.

Let me draw the attention of the reader to the fact that the noncommutative spectral geometry approach discussed here, goes beyond the noncommutative geometry notion employed in the literature to implement the fuzziness of space-time by means of $\left[\mathbf{x}^{i}, \mathbf{x}^{j}\right]=i \theta^{i j}$, where $\theta^{i j}$ is an antisymmetric, real, $d \times d$ ( $d$ is the dimension of space-time) matrix, and $\mathbf{x}^{i}$ denote spatial coordinates.

\section{Elements of Noncommutative Spectral Geometry}

To extend the Riemanian paradigm of geometry to the notion of metric on a noncommutative space, the latter should contain the Riemanian manifold with the metric tensor (as a special case), allow for departures from commutativity of coordinates as well as for quantum corrections of geometry, contain spaces of complex dimension, and offer the means of expressing the Standard Model coupled to Einstein gravity as pure gravity on a suitable geometry. Noncommutative spectral geometry considers the SM as a phenomenological model, which should however determine the geometry of space-time, so that the Maxwell-Dirac action functional leads to the SM action. The geometrical space turns out to be the product of a continuum manifold for the space-time geometry and a noncommutative space for the internal geometry of the SM.

Adopting the simplest generalisation beyond commutative spaces, we consider the geometry of space-time to be described by the tensor product $\mathscr{M} \times \mathscr{F}$ of a continuum compact Riemanian manifold $\mathscr{M}$ and a tiny discrete finite noncommutative space $\mathscr{F}$ composed of just two points. It is worth noting that while the metric dimension of $\mathscr{F}$ is zero, its $K$-theoretic dimension is equal to 6 modulo 8. Following this prescription, the Lagrangian of the SM, including mixing and Majorana mass terms for neutrinos, minimally coupled to gravity is recovered from spectral invariants of the inner fluctuations of the product metric on $\mathscr{M} \times \mathscr{F}$.

The noncommutative nature of the discrete space $\mathscr{F}$ is given by a spectral triple $(\mathscr{A}, \mathscr{H}, \mathscr{D})$, where $\mathscr{A}$ is an involution of operators on the finite-dimensional Hilbert space $\mathscr{H}$ of Euclidean fermions, and $\mathscr{D}$ is a self-adjoint unbounded operator in $\mathscr{H}$. For commutative geometries, the classical notion of a real variable is described as a real-valued function on a space, described by the corresponding algebra $\mathscr{A}$ of coordinates, which for noncommutative geometries is represented as operators in a fixed Hilbert space $\mathscr{H}$. Since real coordinates are represented by self-adjoint operators, all information about a space is encoded in the algebra of coordinates $\mathscr{A}$, which is related to the gauge group of local gauge transformations. By dropping the commutativity property, the infinitesimal line element $d s$, employed to define geometry through the measurement of distances $d(x, y)$ through $d(x, y)=\inf \int_{\gamma} d s$ where the infimum is considered over all possible paths from point $x$ to $y$, does not need to be localised. The absence of commutation of the line element with the coordinates renders possible the measurement of distances through the formula $d(x, y)=\sup \{|f(x)-f(y)|: f \in \mathscr{A},\|\mathscr{D}, f\| \leq 1\}$, where $\mathscr{D}$ denotes the inverse of the line element.

Assuming the algebra $\mathscr{A}$ to be symplectic-unitary, implies $\mathscr{A}=M_{a}(\mathbb{H}) \oplus M_{k}(\mathbb{C})$, with $k=2 a$ and $\mathbb{H}$ denoting the algebra of quaternions, which plays an important rôle here and its choice remains to be explained; we assume quaternion linearity to obtain the SM. The choice $k=4$ is the 
first value that produces the correct number $\left(k^{2}=16\right)$ of fermions in each of the three generations [3], with the number of generations being a physical input. If at the Large Hadron Collider new particles are discovered, one may be able to accommodate them by considering a higher value for $k$. While the choice of algebra $\mathscr{A}$ constitutes the main input of the theory, the choice of Hilbert space $\mathscr{H}$ is irrelevant, since all separable infinite-dimensional Hilbert spaces are isomorphic. The operator $\mathscr{D}$ corresponds to the inverse of the Euclidean propagator of fermions, and is given by the Yukawa coupling matrix which encodes the masses of the elementary fermions and the KobayashiMaskawa mixing parameters. We thus describe geometry by focusing on the Dirac operator $\mathscr{D}$, instead of the metric tensor $g_{\mu \nu}$, used for spaces whose coordinates commute. The fermions of the SM provide the Hilbert space $\mathscr{H}$ of a spectral triple for the algebra $\mathscr{A}$, while the bosons of the SM are obtained through inner fluctuations of the Dirac operator of the product geometry.

All experimental data are of a spectral nature, thus our aim is to extract information, from our noncommutative geometry construction, which is of a spectral nature. Luckily the appropriate tool in noncommutative geometry has been developed; the Spectral Action Functional in noncommutative spaces is the analogous to the Fourier Transform used in spaces characterised by commutation of the coordinates. To obtain the full Lagrangian of the SM, minimally coupled to gravity, we will apply the Spectral Action Principle, stating that the bosonic part of the spectral action functional depends only on the spectrum of the Dirac operator and its asymptotic expression, and for large energy $\Lambda$ is of the form $\operatorname{Tr}(f(\mathscr{D} / \Lambda))$, with $f$ being a cut-off function, whose choice plays only a small rôle; both $\mathscr{D}$ and $\Lambda$ have physical dimensions of a mass and there is no absolute scale on which they can be measured. The rôle of the cut-off scale $\Lambda$ is equivalent to keeping only frequencies smaller than the mass scale $\Lambda$. According to the spectral action principle, $\operatorname{Tr}(f(\mathscr{D} / \Lambda))$ is the fundamental action functional that can be used not only at the classical level but also at the quantum level, after Wick rotation to Euclidean signature. The cut-off-dependent Euclidean action is viewed (in the Wilsonian approach) as the bare action at mass scale $\Lambda$. The physical Lagrangian has also a fermionic part, which has the simple linear form $(1 / 2)\langle J \psi, \mathscr{D} \psi\rangle$, where $J$ is the real structure on the spectral triple and $\psi$ are spinors defined on the Hilbert space. To study cosmological implications of this gravitational model one may consider only the bosonic part of the action; the fermionic part is important to deduce particle physics phenomenology.

The formalism of spectral triples favours Euclidean rather than Lorentzian signature. The discussion of phenomenological aspects of the theory relies on a Wick rotation to imaginary time, into the Lorentzian signature. While sensible from the phenomenological point of view, there exists as yet no justification on the level of the underlying theory. It is worth noting however that the issue of Euclidean versus Lorentzian signature is not a kind of pathology only for the case of noncommutative spectral geometry, it is for instance encountered in the nonperturbative pathintegral approach to quantum gravity.

Applying the spectral action principle to the inner fluctuations of the product $\mathscr{M} \times \mathscr{F}$ of an ordinary compact spin 4-manifold with the finite noncommutative geometry, one recovers the Standard Model action coupled to Einstein and Weyl gravity plus higher order nonrenormalisable interactions suppressed by powers of the inverse of the mass scale of the theory [4]. This model provides specific values of some of the SM parameters at unification scale (denoted by $\Lambda$ ). Following the Wilsonian approach, one can then obtain physical predictions for the SM parameters by running them down to low (present) energy scales through the Renormalisation Group Equations (RGE). 
Using heat kernel methods, the trace $\operatorname{Tr}(f(\mathscr{D} / \Lambda))$ can be written in terms of the geometrical Seeley - de Witt coefficients $a_{n}$, which are known for any second order elliptic differential operator, as $\sum_{n=0}^{\infty} F_{4-n} \Lambda^{4-n} a_{n}$, where the function $F$ is defined such that $F\left(\mathscr{D}^{2}\right)=f(\mathscr{D})$. Thus, the bosonic part of the spectral action can be expanded in powers of $\Lambda$ in the form $[5,6]$

$$
\operatorname{Tr}\left(f\left(\frac{\mathscr{D}}{\Lambda}\right)\right) \sim \sum_{k \in \operatorname{DimSp}} f_{k} \Lambda^{k} f|\mathscr{D}|^{-k}+f(0) \zeta_{\mathscr{D}(0)}+\mathscr{O}(1) .
$$

The momenta $f_{k}$ are defined as $f_{k} \equiv \int_{0}^{\infty} f(u) u^{k-1} \mathrm{~d} u$ for $k>0$ and $f_{0} \equiv f(0)$, the noncommutative integration is defined in terms of residues of zeta functions $\zeta_{\mathscr{D}}(s)=\operatorname{Tr}\left(|\mathscr{D}|^{-s}\right)$ at poles of the zeta function, and the sum is over points in the dimension spectrum of the spectral triple.

Considering the Riemanian geometry to be four-dimensional, the asymptotic expansion of the trace reads

$$
\operatorname{Tr}\left(f\left(\frac{D}{\Lambda}\right)\right) \sim 2 \Lambda^{4} f_{4} a_{0}+2 \Lambda^{2} f_{2} a_{2}+f_{0} a_{4}+\cdots+\Lambda^{-2 k} f_{-2 k} a_{4+2 k}+\cdots
$$

The smooth even function $f$, which decays fast at infinity, only enters in the multiplicative factors:

$$
\begin{aligned}
f_{4}=\int_{0}^{\infty} f(u) u^{3} d u \quad, \quad f_{2}=\int_{0}^{\infty} f(u) u d u, \\
f_{0}=f(0) \quad, \quad f_{-2 k}=(-1)^{k} \frac{k !}{(2 k) !} f^{(2 k)}(0) .
\end{aligned}
$$

Since $f$ is taken as a cut-off function, its Taylor expansion at zero vanishes, thus its asymptotic expansion, Eq. (2.2), reduces to just

$$
\operatorname{Tr}\left(f\left(\frac{D}{\Lambda}\right)\right) \sim 2 \Lambda^{4} f_{4} a_{0}+2 \Lambda^{2} f_{2} a_{2}+f_{0} a_{4} .
$$

The cut-off function enters through its momenta $f_{0}, f_{2}, f_{4}$; these three additional real parameters are physically related to the coupling constants at unification, the gravitational constant and the cosmological constant. In the four-dimensional case, the term in $\Lambda^{4}$ in the spectral action, Eq. (2.1), gives a cosmological term, the term in $\Lambda^{2}$ gives the Einstein-Hilbert action functional with the physical sign for the Euclidean functional integral (provided $f_{2}>0$ ), and the $\Lambda$-independent term yields the Yang-Mills action for the gauge fields corresponding to the internal degrees of freedom of the metric. The scale-independent terms in the spectral action have conformal invariance. Note that the arbitrary mass scale $\Lambda$ can be made dynamical by introducing a scaling dilaton field.

Since the physical Lagrangian is entirely determined by the geometric input, the physical implications of this approach are closely dependent on the underlying chosen geometry. The obtained physical Lagrangian contains, in addition to the full Standard Model Lagrangian, the EinsteinHilbert action with a cosmological term, a topological term related to the Euler characteristic of the space-time manifold, a conformal Weyl term and a conformal coupling of the Higgs field to gravity. The bosonic action in Euclidean signature reads [4]

$$
\begin{array}{r}
\mathscr{S}^{\mathrm{E}}=\int\left(\frac{1}{2 \kappa_{0}^{2}} R+\alpha_{0} C_{\mu v \rho \sigma} C^{\mu v \rho \sigma}+\gamma_{0}+\tau_{0} R^{\star} R^{\star}+\frac{1}{4} G_{\mu \nu}^{i} G^{\mu v i}+\frac{1}{4} F_{\mu \nu}^{\alpha} F^{\mu v \alpha}\right. \\
\left.+\frac{1}{4} B^{\mu v} B_{\mu \nu}+\frac{1}{2}\left|D_{\mu} \mathbf{H}\right|^{2}-\mu_{0}^{2}|\mathbf{H}|^{2}-\xi_{0} R|\mathbf{H}|^{2}+\lambda_{0}|\mathbf{H}|^{4}\right) \sqrt{g} d^{4} x,
\end{array}
$$


where

$$
\begin{aligned}
\kappa_{0}^{2}=\frac{12 \pi^{2}}{96 f_{2} \Lambda^{2}-f_{0} \mathfrak{c}} \quad, \quad \alpha_{0}=-\frac{3 f_{0}}{10 \pi^{2}}, \\
\gamma_{0}=\frac{1}{\pi^{2}}\left(48 f_{4} \Lambda^{4}-f_{2} \Lambda^{2} \mathfrak{c}+\frac{f_{0}}{4} \mathfrak{d}\right) \quad, \quad \tau_{0}=\frac{11 f_{0}}{60 \pi^{2}} \\
\mu_{0}^{2}=2 \Lambda^{2} \frac{f_{2}}{f_{0}}-\frac{\mathfrak{e}}{\mathfrak{a}}, \quad \xi_{0}=\frac{1}{12} \quad, \quad \lambda_{0}=\frac{\pi^{2} \mathfrak{b}}{2 f_{0} \mathfrak{a}^{2}}
\end{aligned}
$$

$\mathbf{H}$ is a rescaling $\mathbf{H}=\left(\sqrt{a f_{0}} / \pi\right) \phi$ of the Higgs field $\phi$ to normalize the kinetic energy, and the momentum $f_{0}$ is physically related to the coupling constants at unification. Notice the absence of quadratic terms in the curvature; there is only the term quadratic in the Weyl curvature and the topological term $R^{\star} R^{\star}$. In a cosmological setting, namely for Friedmann-Lemaitre-RobertsonWalker (FLRW) geometries, the Weyl term vanishes. Notice also the term that couples gravity with the SM, a term which should always be present when one considers gravity coupled to scalar fields.

Writing the asymptotic expansion of the spectral action, a number of geometric parameters appeared, which describe the possible choices of Dirac operators on the finite noncommutative space. These parameters correspond to the Yukawa parameters of the particle physics model and the Majorana terms for the right-handed neutrinos. They are given by [4]

$$
\begin{aligned}
\mathfrak{a} & =\operatorname{Tr}\left(Y_{(\uparrow 1)}^{\star} Y_{(\uparrow 1)}+Y_{(\downarrow 1)}^{\star} Y_{(\downarrow 1)}+3\left(Y_{(\uparrow 3)}^{\star} Y_{(\uparrow 3)}+Y_{(\downarrow 3)}^{\star} Y_{(\downarrow 3)}\right)\right), \\
\mathfrak{b} & =\operatorname{Tr}\left(\left(Y_{(\uparrow 1)}^{\star} Y_{(\uparrow 1)}\right)^{2}+\left(Y_{(\downarrow 1)}^{\star} Y_{(\downarrow 1)}\right)^{2}+3\left(Y_{(\uparrow 3)}^{\star} Y_{(\uparrow 3)}\right)^{2}+3\left(Y_{(\downarrow 3)}^{\star} Y_{(\downarrow 3)}\right)^{2}\right), \\
\mathfrak{c} & =\operatorname{Tr}\left(Y_{R}^{\star} Y_{R}\right), \\
\mathfrak{d} & =\operatorname{Tr}\left(\left(Y_{R}^{\star} Y_{R}\right)^{2}\right), \\
\mathfrak{e} & =\operatorname{Tr}\left(Y_{R}^{\star} Y_{R} Y_{(\uparrow 1)}^{\star} Y_{(\uparrow 1)}\right),
\end{aligned}
$$

with $Y_{(\downarrow 1)}, Y_{(\uparrow 1)}, Y_{(\downarrow 3)}, Y_{(\uparrow 3)}$ and $Y_{R}$ being $(3 \times 3)$ matrices, with $Y_{R}$ symmetric. The $Y$ matrices are used to classify the action of the Dirac operator and give the fermion and lepton masses, as well as lepton mixing, in the asymptotic version of the spectral action. The Yukawa parameters run with the RGE of the particle physics model. Since running towards lower energies implies that nonperturbative effects in the spectral action cannot be any longer neglected, any results based on the asymptotic expansion and on renormalisation group analysis can only hold for early universe cosmology. Hence, the spectral action at the unification scale $\Lambda$ offers a framework to investigate early universe cosmological models $[7,8,9,10,11,12]$. For later times, one should consider the full spectral action, a direction which requires the development of nontrivial mathematical tools.

It is important to emphasise that the relations given in Eq. (2.6) above are tied to the scale at which the expansion is performed. There is a priori no reason for the constraints to hold at scales below the unification scale $\Lambda$, since they represent mere boundary conditions. One should therefore be very careful and keep in mind that it is incorrect to consider the relations in Eq. (2.6) as functions of the energy scale; these relations are only valid at unification scale $\Lambda$. 


\section{High Energy Phenomenology of the Noncommutative Spectral Geometry}

In what follows we assume that the function $f$ is well approximated by the cut-off function and ignore higher order terms. Normalisation of the kinetic terms implies

$$
\frac{g_{3}^{2} f_{0}}{2 \pi^{2}}=\frac{1}{4} \text { and } g_{3}^{2}=g_{2}^{2}=\frac{5}{3} g_{1}^{2}
$$

while

$$
\sin ^{2} \theta_{\mathrm{W}}=\frac{3}{8}
$$

a relation which was also found for $\mathrm{SU}(5)$ and $\mathrm{SO}(10)$. Assuming the big desert hypothesis, the running of the couplings $\alpha_{i}=g_{i}^{2} /(4 \pi)$ with $i=1,2,3$ can then be obtained via the RGE.

The phenomenological consequences of the noncommutative spectral geometry as an approach to unification have been discussed in Ref. [4], where the authors considered only one-loop corrections, for which the $\beta$-functions are $\beta_{g_{i}}=(4 \pi)^{-2} b_{i} g_{i}^{3}$ with $i=1,2,3$ and $b=(41 / 6,-19 / 6,-7)$. It is worth noticing that only at one-loop order the Renormalisation Group Equations for the coupling constants $g_{i}$ are uncoupled from the other Standard Model parameters.

Performing one-loop RGE for the running of the gauge couplings and the Newton constant, it was shown [4] that these do not meet at a point, the error being within just few percent. The fact that the predicted unification of the coupling constants does not hold exactly, implies that the big desert hypothesis is only approximately valid and new physics are expected between unification and present energy scales. In terms of our assumption for the cut-off function, the lack of a unique unification energy, implies that even though the function $f$ can be approximated by the cut-off function, there exist small deviations. Besides this result it is however worth noting that the model leads to the correct representations of the fermions with respect to the gauge group of the SM, the Higgs doublet appears as part of the inner fluctuations of the metric, and Spontaneous Symmetry Breaking mechanism arises naturally with the negative mass term without any tuning. In addition, the see-saw mechanism is obtained, the 16 fundamental fermions are recovered, and a top quark mass of $M_{\text {top }} \sim 179 \mathrm{GeV}$ is predicted.

The model predicts a heavy Higgs mass; in zeroth order approximation, it predicts a mass of the Higgs boson approximately equal to $170 \mathrm{GeV}$, which strictly speaking is ruled out by current experimental data. Due to this discrepancy between the NCG prediction and the experimental data, noncommutative spectral geometry has been (rather unfairly) criticised, even though the result quoted above depends on the value of the gauge couplings at unification scale, which is uncertain and was obtained neglecting the nonminimal coupling between the Higgs field and the Ricci curvature. I believe that one should instead draw the conclusion that noncommutative spectral geometry as an approach to unification, even in its present (and certainly simple) version, it still led to the correct order of magnitude for the Higgs mass, a result which was by no means obvious.

Considering an energy scale $\Lambda \sim 1.1 \times 10^{17} \mathrm{GeV}$, the standard form of the gravitational action and the experimental value of Newton's constant at ordinary scales imply $\kappa_{0}^{-1} \sim 2.43 \times 10^{18} \mathrm{GeV}$.

Finally, this approach to unification does not provide any explanation of the number of generations, nor leads to constraints on the values of the Yukawa couplings. 


\section{Cosmological Consequences of the Noncommutative Spectral Geometry}

The Lorentzian version of the gravitational part of the asymptotic formula for the bosonic sector of the action obtained within noncommutative spectral geometry reads [4]

$$
\mathscr{S}_{\text {grav }}^{\mathrm{L}}=\int\left(\frac{1}{2 \kappa_{0}^{2}} R+\alpha_{0} C_{\mu v \rho \sigma} C^{\mu v \rho \sigma}+\tau_{0} R^{\star} R^{\star} \xi_{0} R|\mathbf{H}|^{2}\right) \sqrt{-g} d^{4} x .
$$

This action leads to the following equations of motion [7]

$$
R^{\mu v}-\frac{1}{2} g^{\mu v} R+\frac{1}{B^{2}} \delta_{\mathrm{cc}}\left[2 C_{; \lambda ; \kappa}^{\mu \lambda \nu \kappa}+C^{\mu \lambda v \kappa} R_{\lambda \kappa}\right]=\kappa_{0}^{2} \delta_{\mathrm{cc}} T_{\mathrm{matter}}^{\mu \nu}
$$

where we have introduced $B^{2} \equiv-\left(4 \kappa_{0}^{2} \alpha_{0}\right)^{-1}$, related to the $f_{0}$ moment of the cut-off function and we have captured the nonminimal coupling between the Higgs field and the Ricci curvature scalar in the parameter $\delta_{\mathrm{cc}}$, defined as $\delta_{\mathrm{cc}} \equiv\left[1-2 \kappa_{0}^{2} \xi_{0} \mathbf{H}^{2}\right]^{-1}$.

In the low energy weak curvature regime, the nonminimal coupling between the background geometry and the Higgs field can be neglected, thus $\delta_{\mathrm{cc}}=1$. For a FLRW space-time, the Weyl tensor vanishes, hence the NCG corrections to the Einstein equation vanish [7], rending difficult to restrict $B$ via cosmology or solar-system tests. Imposing a lower limit on $B$ would imply an upper limit to the moment $f_{0}$, and thus restrict particle physics at unification.

One can impose an upper limit to the moment $f_{0}$, by studying the energy lost to gravitational radiation by orbiting binaries $[11,12]$. Considering linear perturbations around a Minkowski background metric, the equations of motion read [12]

$$
\left(\square-B^{2}\right) \square h^{\mu v}=B^{2} \frac{16 \pi G}{c^{4}} T_{\mathrm{matter}}^{\mu \nu},
$$

where $T_{\text {matter }}^{\mu \nu}$ is taken to lowest order in $h^{\mu v}$. Since $B$ plays the rôle of a mass, it must be real and positive, thus $\alpha_{0}$ must be negative for Minkowski space to be a stable vacuum of the theory.

Consider the energy lost to gravitational radiation by orbiting binaries. In the far field limit, $|\mathbf{r}| \approx\left|\mathbf{r}-\mathbf{r}^{\prime}\right|$ (r and $\mathbf{r}^{\prime}$ denote the locations of observer and emitter, respectively), the spatial components of the general first order solution for a perturbation against a Minkowski background read [12]

$$
h^{i k}(\mathbf{r}, t) \approx \frac{2 G B}{3 c^{4}} \int_{-\infty}^{t-\frac{1}{c}|\mathbf{r}|} \frac{d t^{\prime}}{\sqrt{c^{2}\left(t-t^{\prime}\right)^{2}-|\mathbf{r}|^{2}}} \mathscr{J}_{1}\left(B \sqrt{c^{2}\left(t-t^{\prime}\right)^{2}-|\mathbf{r}|^{2}}\right) \ddot{D}^{i k}\left(t^{\prime}\right)
$$

$D^{i k}$ is the quadrupole moment, defined as $D^{i k}(t) \equiv \frac{3}{c^{2}} \int x^{i} x^{k} T^{00}(\mathbf{r}, t) d \mathbf{r}$, and $\mathscr{J}_{1}$ a Bessel function of the first kind. While for $B \rightarrow \infty$ the theory reduces to GR, for finite $B$ gravitational radiation contains massive and massless modes, both sourced from the quadrupole moment of the system.

For a binary pair of masses $m_{1}, m_{2}$ in circular orbit in the $(x y)$-plane, the rate of energy loss is

$$
-\frac{\mathrm{d} \mathscr{E}}{\mathrm{d} t} \approx \frac{c^{2}}{20 G}|\mathbf{r}|^{2} \dot{h}_{i j} \dot{h}^{i j}
$$

with [12]

$$
\dot{h}^{i j} \dot{h}_{i j}=\frac{128 \mu^{2}|\rho|^{4} \omega^{6} G^{2} B^{2}}{c^{8}} \times\left[f_{\mathrm{c}}^{2}\left(B|\mathbf{r}|, \frac{2 \omega}{B c}\right)+f_{\mathrm{s}}^{2}\left(B|\mathbf{r}|, \frac{2 \omega}{B c}\right)\right]
$$




$$
\begin{aligned}
& f_{\mathrm{s}}(x, z) \equiv \int_{0}^{\infty} \frac{d s}{\sqrt{s^{2}+x^{2}}} \mathscr{J}_{1}(s) \sin \left(z \sqrt{s^{2}+x^{2}}\right) \\
& f_{\mathrm{c}}(x, z) \equiv \int_{0}^{\infty} \frac{d s}{\sqrt{s^{2}+x^{2}}} \mathscr{J}_{1}(s) \cos \left(z \sqrt{s^{2}+x^{2}}\right) .
\end{aligned}
$$

The orbital frequency $\omega$, defined in terms of the magnitude $|\rho|$ of the separation vector between the two bodies, is constant and equal to $\omega=|\rho|^{-3 / 2} \sqrt{G\left(m_{1}+m_{2}\right)}$.

The integrals in Eqs. (4.6), (4.7), exhibit a strong resonance behavior at $z=1$, which corresponds to the critical frequency [12]

$$
2 \omega_{\mathrm{c}}=B c
$$

around which strong deviations from the GR results are expected. This maximum frequency results from the natural length scale, given by $B^{-1}$, at which NCG effects become dominant.

There are several binary pulsars for which the rate of change of the orbital frequency has been well characterised, and the predictions of General Relativity agree with the data to a high accuracy. Requiring the magnitude of deviations from GR obtained in the context of noncommutative spectral geometry, to be less than the allowed uncertainty in the data, one constrains $B$, namely [11]

$$
B>7.55 \times 10^{-13} \mathrm{~m}^{-1} \text {. }
$$

This observational constraint may seem weak, however it is comparable to existing constraints on similar, ad hoc, additions to GR. In particular, constraints on additions to the Einstein-Hilbert action, of the form $R^{2}$ and $R_{\mu v} R^{\mu v}$, are of the order of $B_{R^{2}} \geq 3.2 \times 10^{-9} \mathrm{~m}^{-1}$, where $B_{R^{2}}$ is the $B$ parameter associated with the couplings of these terms [13]. Note also that since the strongest constraint comes from systems with high orbital frequencies, future observations of rapidly orbiting binaries, relatively close to the Earth, could improve it by many orders of magnitude.

Remaining in the low-energy limit, in other words neglecting the coupling between the Higgs field and the background geometry, on may consider the corrections to the background Einstein's equations. It turns out that noncommutative corrections do not occur at the level of a FLRW background, since then the modified Friedmann equation reduces to its standard form [7]. One may have naively claimed that this was expected, arguing that in a spatially homogeneous spacetime the spatial points are equivalent and any noncommutative effects are then expected to vanish. However, this argument does not apply here, since the noncommutativity is incorporated in the internal manifold $\mathscr{F}$ and the space-time is a commutative four-dimensional manifold.

Neglecting the nonminimal coupling between the Higgs field and the Ricci curvature, any modifications to the background equation will be apparent at leading order for anisotropic and inhomogeneous models. Let us consider the representative example of Bianchi type-V model, for which the space-time metric, in Cartesian coordinates, reads

$$
g_{\mu \nu}=\operatorname{diag}\left[-1,\left\{a_{1}(t)\right\}^{2} e^{-2 n z},\left\{a_{2}(t)\right\}^{2} e^{-2 n z},\left\{a_{3}(t)\right\}^{2}\right] ;
$$

$a_{i}(t)$ with $i=1,2,3$ arbitrary functions and $n$ is an integer. 
Defining $A_{i}(t)=\ln a_{i}(t)$ with $i=1,2,3$, the modified Friedmann equation reads [7]:

$$
\begin{array}{r}
\kappa_{0}^{2} T_{00}= \\
-\dot{A}_{3}\left(\dot{A}_{1}+\dot{A}_{2}\right)-n^{2} e^{-2 A_{3}}\left(\dot{A}_{1} \dot{A}_{2}-3\right) \\
+\frac{8 \alpha_{0} \kappa_{0}^{2} n^{2}}{3} e^{-2 A_{3}}\left[5\left(\dot{A}_{1}\right)^{2}+5\left(\dot{A}_{2}\right)^{2}-\left(\dot{A}_{3}\right)^{2}-\dot{A}_{1} \dot{A}_{2}-\dot{A}_{2} \dot{A}_{3}-\dot{A}_{3} \dot{A}_{1}-\ddot{A}_{1}-\ddot{A}_{2}-\ddot{A}_{3}+3\right] \\
-\frac{4 \alpha_{0} \kappa_{0}^{2}}{3} \sum_{i}\left\{\dot{A}_{1} \dot{A}_{2} \dot{A}_{3} \dot{A}_{i}+\dot{A}_{i} \dot{A}_{i+1}\left(\left(\dot{A}_{i}-\dot{A}_{i+1}\right)^{2}-\dot{A}_{i} \dot{A}_{i+1}\right)\right. \\
+\left(\ddot{A}_{i}+\left(\dot{A}_{i}\right)^{2}\right)\left[-\ddot{A}_{i}-\left(\dot{A}_{i}\right)^{2}+\frac{1}{2}\left(\ddot{A}_{i+1}+\ddot{A}_{i+2}\right)+\frac{1}{2}\left(\left(\dot{A}_{i+1}\right)^{2}+\left(\dot{A}_{i+2}\right)^{2}\right)\right] \\
\left.+\left[\dddot{A}_{i}+3 \dot{A}_{i} \ddot{A}_{i}-\left(\ddot{A}_{i}+\left(\dot{A}_{i}\right)^{2}\right)\left(\dot{A}_{i}-\dot{A}_{i+1}-\dot{A}_{i+2}\right)\right]\left[2 \dot{A}_{i}-\dot{A}_{i+1}-\dot{A}_{i+2}\right]\right\}(4
\end{array}
$$

with $i=1,2,3$, while the $t$-dependence of the terms has been omitted for simplicity.

Any term containing $\alpha_{0}$ in Eq. (4.11), encodes a modification from the standard result. Let us study Eq. (4.11) above. The correction terms can be divided into two types. The first one contains the terms in braces in Eq. (4.11), which are fourth order in time derivatives. Thus, for the slowly varying functions, usually considered in cosmology, these corrections can be neglected. The second type, which appears in the third line in Eq. (4.11), occurs at the same order as the standard Einstein-Hilbert terms. However, since this term is proportional to $n^{2}$, it vanishes for homogeneous versions of Bianchi type-V. Thus, although anisotropic cosmologies do contain corrections due to the additional NCG terms in the action, they are typically of higher order [7]. Inhomogeneous models do contain correction terms that appear on the same footing as the original terms.

It is worth noting that by studying the case of the Bianchi type V model, one can identify the noncommutative geometry effects in other cases of cosmological models, for instance Bianchi I and Kasner models. In conclusion, the corrections to Einstein's equations can only be important for inhomogeneous and anisotropic space-times [7].

Certainly, the coupling between the Higgs field and the background geometry cannot be neglected once the energies reach the Higgs scale. The nonminimal coupling of Higgs field to curvature leads to corrections to Einstein's equations even for homogeneous and isotropic cosmological models. To illustrate the effects of these corrections let us neglect the conformal term in Eq. (4.2), so that the equations of motion read [7]

$$
R^{\mu v}-\frac{1}{2} g^{\mu v} R=\kappa_{0}^{2}\left[\frac{1}{1-\kappa_{0}^{2}|\mathbf{H}|^{2} / 6}\right] T_{\text {matter }}^{\mu v},
$$

implying that $|\mathbf{H}|$ plays the rôle of an effective gravitational constant [7].

The nonminimal coupling between the Higgs field and the Ricci curvature may turn out to be crucial in early universe cosmology $[8,10]$. Such a coupling has been introduced ad hoc in the literature, in an attempt to drive inflation through the Higgs field, and thus cure one of the main pathologies of the inflationary paradigm, namely the origin of the inflaton field. However, the value of the coupling constant between the scalar field and the background geometry should be dictated by the underlying theory. Actually, even if classically the coupling between the Higgs field and the 
Ricci curvature could be set equal to zero, a nonminimal coupling will be induced once quantum corrections in the classical field theory are considered.

In a FLRW metric, the Gravity-Higgs sector of the asymptotic expansion of the spectral action, in Lorentzian signature, reads

$$
S_{\mathrm{GH}}^{\mathrm{L}}=\int\left[\frac{1-2 \kappa_{0}^{2} \xi_{0} H^{2}}{2 \kappa_{0}^{2}} R-\frac{1}{2}(\nabla H)^{2}-V(H)\right] \sqrt{-g} d^{4} x,
$$

where

$$
V(H)=\lambda_{0} H^{4}-\mu_{0}^{2} H^{2},
$$

with $\mu_{0}$ and $\lambda_{0}$ subject to radiative corrections as functions of energy. For large enough values of the Higgs field, the renormalised value of $\mu_{0}$ and $\lambda_{0}$ must be calculated. More precisely, one takes the measured values of the gauge couplings at low energy and using $\beta$-functions one evolves them in higher energy scales, taking into account the thresholds where quark species come into the running. Note that one should evolve simultaneously the running of the top Yukawa coupling and the gauge couplings.

At high energies the mass term in Eq. (4.14) is sub-dominant and can be neglected. As an explicit analysis has shown [10], for each value of the top quark mass there is a value of the Higgs mass where the effective potential is about to develop a metastable minimum at large values of the Higgs field and the Higgs potential is locally flattened. Calculating [10] the renormalisation of the Higgs self-coupling up to two-loops, we have constructed an effective potential which fits the renormalisation group improved potential around the flat region. We have found [10] a very good analytic fit to the Higgs potential around the minimum of the potential:

$$
V^{\mathrm{eff}}=\lambda_{0}^{\mathrm{eff}}(H) H^{4}=\left[a \ln ^{2}(b \kappa H)+c\right] H^{4},
$$

where the parameters $a, b$ are related to the low energy values of top quark mass $m_{\mathrm{t}}$ as [10]

$$
\begin{aligned}
& a\left(m_{\mathrm{t}}\right)=4.04704 \times 10^{-3}-4.41909 \times 10^{-5}\left(\frac{m_{\mathrm{t}}}{\mathrm{GeV}}\right)+1.24732 \times 10^{-7}\left(\frac{m_{\mathrm{t}}}{\mathrm{GeV}}\right)^{2}, \\
& b\left(m_{\mathrm{t}}\right)=\exp \left[-0.979261\left(\frac{m_{\mathrm{t}}}{\mathrm{GeV}}-172.051\right)\right] .
\end{aligned}
$$

The third parameter, $c$, encodes the appearance of an extremum and depends on the values for top quark mass and Higgs mass. An extremum occurs if and only if $c / a \leq 1 / 16$, the saturation of the bound corresponding to a perfectly flat region. It is convenient to write $c=[(1+\delta) / 16] a$, where $\delta=0$ saturates the bound below which a local minimum is formed.

This study was done in the case of minimal coupling, while the modifications for $\xi_{0}=1 / 12$ imply that flatness does not occur at $\delta=0$, but for fixed values of $\delta$ depending on the value of the top quark mass. Hence, for inflation to occur via the Higgs field, the top quark mass fixes the Higgs mass extremely accurately.

Since the region where the potential is flat is narrow, to achieve a long enough period of quasi-exponential expansion, requires that slow-roll must be indeed very slow. Thus, the slowroll parameters, $\varepsilon$ and $\eta$ must be slow enough to allow sufficient number of e-folds. In addition, the amplitude of density perturbations $\Delta_{\mathscr{R}}^{2}$ in the Cosmic Microwave Background (CMB) must be within the window allowed from the most recent experimental data. More precisely, inflation 
predicts that at horizon crossing (denoted by stars), the amplitude of density perturbations is related to the inflaton potential through $\left(V_{*} / \varepsilon_{*}\right)^{1 / 4}=2 \sqrt{3 \pi} m_{\mathrm{Pl}} \Delta_{\mathscr{R}}^{1 / 2}$, where $\varepsilon_{*} \leq 1$. Its value, as measured by WMAP7 [14], requires $\left(V_{*} / \varepsilon_{*}\right)^{1 / 4}=(2.75 \pm 0.30) \times 10^{-2} m_{\mathrm{Pl}}$, where $m_{\mathrm{Pl}}$ is the Planck mass.

A systematic search in the parameter space was performed in Ref. [10] using a Monte-Carlo chain. It was found that even though slow-roll inflation can be realised - a result which does not hold for minimally coupled Higgs field - the resulting ratio of perturbation amplitudes is too large for any experimentally allowed values for the masses of the top quark and the Higgs boson. Hence, Higgs driven inflation cannot be accommodated in the noncommutative spectral geometry model studied here, a result which coincides with the conclusion known for ordinary commutative geometries. It is worth noting that running of the gravitational constant and corrections by considering the more appropriate de Sitter space-time, instead of the Minkowski geometry employed here, do not improve substantially the realisation of a successful slow-roll inflationary era [10].

The NCG Spectral Action provides, in addition to the Higgs field, another conformally coupled (massless) scalar field, which exhibits no coupling to the matter sector [15]. Including this field, the cosmologically relevant terms in the Wick rotated action read

$$
S^{\mathrm{L}}=\int\left\{\frac{1}{2 \kappa^{2}} R-\xi_{\mathbf{H}} R \mathbf{H}^{2}-\xi_{\sigma} R \sigma^{2}-\frac{1}{2}(\nabla \mathbf{H})^{2}-\frac{1}{2}(\nabla \sigma)^{2}-V(\mathbf{H}, \sigma)\right\} \sqrt{-g} d^{4} x,
$$

where

$$
V(\mathbf{H}, \sigma)=\lambda_{\mathbf{H}} \mathbf{H}^{4}-\mu_{\mathbf{H}}^{2} \mathbf{H}^{2}+\lambda_{\sigma} \sigma^{4}+\lambda_{\mathbf{H} \sigma}|\mathbf{H}|^{2} \sigma^{2} .
$$

The constants are related to the underlying parameters as

$$
\xi_{\mathbf{H}}=\frac{1}{12}, \quad \xi_{\sigma}=\frac{1}{12}, \lambda_{\mathbf{H}}=\frac{\pi^{2} \mathfrak{b}}{2 f_{0} \mathfrak{a}^{2}}, \lambda_{\sigma}=\frac{\pi^{2} \mathfrak{d}}{f_{0} \mathfrak{c}^{2}}, \quad \mu_{\mathbf{H}}=2 \Lambda^{2} \frac{f_{2}}{f_{0}}, \lambda_{\mathbf{H} \sigma}=\frac{2 \pi^{2} \mathfrak{e}}{a \mathfrak{c} f_{0}} .
$$

A careful analysis performed in Ref. [10] has shown that neither this field can lead to a successful slow-roll inflationary era if the coupling values are conformal.

It is worth noting that the above conclusions may alter if $\xi_{0}=1 / 12$ turns out not to be a generic feature of noncommutative geometries. However, let me emphasise that there are no nonconformal values for the coupling $\xi_{0}$ for which there is a renormalization group flow towards the conformal value as one runs the Standard Model parameters up in the energy scale. This implies that if one expects an exactly conformal coupling for the Higgs field at some specific scale, it will be exactly conformal at all scales.

\section{Outlook and Conclusions}

Noncomutative spectral geometry provides an elegant way of expressing the full Standard Model of strong and electroweak interactions coupled to Einstein gravity, as pure gravity on a modified space-time geometry. The paradigm of metric noncommutative geometry studied here is of a spectral nature, an important notion in physics since all experimental data are indeed of a spectral type. This approach is fundamentally different than any other paradigm, which imposes a particular structure for geometrical spaces in the quantum gravity regime. Connes' model focuses on an almost commutative space, considering that at energies close but lower than Planckian energy scales, space can be described by the tensor product of a continuum manifold by a discrete space. 
Within the context of noncommutative spectral geometry, gravity and matter are treated in a similar way, leading to concrete relationships between matter and gravitational couplings. The asymptotic expansion of the gravitational sector of the theory leads to modifications to General Relativity which can be used to constrain the theory through astrophysical observations. Considering the energy lost by binary systems to gravitational radiation, we were able to restrict the value of the Weyl squared coupling in the bosonic action.

Investigating Higgs driven inflation within noncomutative spectral action, we have shown that while the Higgs potential can lead to the slow-roll conditions being satisfied once the running of the self-coupling at two-loops is included, the constraints imposed from the CMB data make the predictions of such a scenario incompatible with the measured value of the top quark mass. Another massless scalar field, which naturally appears in the model, seems also not to lead to a successful era of slow-roll inflation. However, the arbitrary mass scale $\Lambda$ in the spectral action for the Dirac operator can be made dynamical by introducing a dilaton field; this dilaton field may turn out to be a successful inflaton candidate.

Noncommutative spectral geometry faces, to my opinion, at least two immediate research directions, essential in order to deduce further cosmological and phenomenological consequences of this paradigm. Firstly, one should compute higher order terms in the asymptotic expansion of the spectral action functional. Note that it is very difficult to compute exactly the spectral action in its nonperturbative form, even though some progress has been made however recently [16]. Since the action functional $\operatorname{Tr}(f(\mathscr{D} / \Lambda))$ is not local - its locality is only achieved when it is replaced by the asymptotic expansion - at least the next term in the asymptotic expansion must be computed, in order to check the validity of the asymptotic expansion. It was recently shown [16] that for a space-time whose spatial sections are 3-spheres $S^{3}$, Wick rotated and compactified to a Euclidean model $S^{3} \times S^{1}$, the spectral action is given, for any test function, by the sum of two terms up to a remarkably tiny correction. Let me emphasise that for any low-energy astrophysical consequence of the noncommutative spectral geometry, a priori the full spectral action, and not only its asymptotic form, has to be considered.

Secondly, it could be of great importance to find the running of the parameters appearing in the spectral action, since otherwise it is impossible to extract information for low-energy astrophysical events. It is worth repeating that the expressions for $\kappa_{0}, \alpha_{0}, \gamma_{0}, \tau_{0}, \mu_{0}, \lambda_{0}$ in terms of $f_{0}, f_{2}, f_{4}, \mathfrak{a}, \mathfrak{b}, \mathfrak{c}, \mathfrak{d}, \mathfrak{e}$ and the conformal value for $\xi_{0}$ are only valid at unification scale $\Lambda$. It is simply incorrect to naively postulate that these equalities can hold at lower energy scales as such, by just considering the parameters $\kappa, \alpha, \cdots$ as functions of the energy scale.

At last but not least, one should consider less trivial noncommutative spaces whose limit is the almost commutative space considered in the original Connes' model discussed here.

Nevertheless, besides these necessary further developments, it is fair to conclude by stating that noncommutative spectral geometry offers a beautiful mathematical construction which provides an elegant explanation for the most successful particle physics model at hand.

It is a pleasure to thank the organisers of the Workshop on Non Commutative Field Theory and Gravity, held in the beautiful island of Corfu, for inviting me to present this work during a stimulating and interesting meeting. 


\section{References}

[1] A. Connes, Noncommutative Geometry, Academic Press, New York (1994).

[2] A. Connes and M. Marcolli, Noncommutative Geometry, Quantum Fields and Motives, Hindustan Book Agency, India (2008).

[3] A. H. Chamseddine and A. Connes, Phys. Rev. Lett. 99, 191601 (2007) [arXiv:0706.3690 [hep-th]].

[4] A. H. Chamseddine, A. Connes and M. Marcolli, Adv. Theor. Math. Phys. 11, 991 (2007) [arXiv:hep-th/0610241].

[5] A. H. Chamseddine and A. Connes, Phys. Rev. Lett. 77, 4868 (1996).

[6] A. H. Chamseddine and A. Connes, Comm. Math. Phys. 186, 731 (1977).

[7] W. Nelson and M. Sakellariadou, Phys. Rev. D 81, 085038 (2010) [arXiv:0812.1657 [hep-th]].

[8] W. Nelson and M. Sakellariadou, Phys. Lett. B 680, 263 (2009) [arXiv:0903.1520 [hep-th]].

[9] M. Marcolli and E. Pierpaoli, arXiv:0908.3683 [hep-th].

[10] M. Buck, M. Fairbairn and M. Sakellariadou, Phys. Rev. D 82043509 (2010) [arXiv:1005.1188 [hep-th]].

[11] W. Nelson, J. Ochoa and M. Sakellariadou, Phys. Rev. Lett. 105 (2010) 101602 [arXiv:1005.4279 [hep-th]].

[12] W. Nelson, J. Ochoa and M. Sakellariadou, Phys. Rev. D 82 (2010) 085021 [arXiv:1005.4276 [hep-th]].

[13] K. S. Steele, Gen. Rel. Grav. 9353 (1978).

[14] D. Larson et al., [arXiv:1001.4758 [astro-ph]].

[15] A. H. Chamseddine, [arXiv:0901.0577 [hep-th]].

[16] A. H. Chamseddine and A. Connes, Comm. Math. Phys. 293, 867 (2010) arXiv:0812.0165 [hep-th]. 